\section{DRAFT DESIGN SOLUTION FOR AUTOMATED 3D SCANNING OF PERSONS}

RADOMIR MENDRICKY, VRATISLAV MALY

Department of Manufacturing Systems and Automation, Technical University of Liberec, Liberec, Czech Republic

DOI : 10.17973/MMSJ.2019_12_2019005

E-mail: radomir.mendricky@tul.cz

This article deals with a process of designing an inexpensive device for automated scanning of people and large objects. The attention is paid to selection of technical solution of individual system components, from construction to calculation for motors of individual movement axes. The result is a device that allows automated digitization of objects with up to two metres of height, using simple and affordable hand-held scanners.

KEYWORDS

Construction design, 3D scanning, digitization, automated scanning of persons, Scanner Sense

\section{INTRODUCTION}

Digitization is an indispensable part of modern age, finding its use in large scale of operations and offering new possibilities to mankind. Specific fields that use 3D scanning are for example medicine or textile industry. Both these fields are primarily based on human skill, however, digitization pushes it to the next level.

The goal of this project is to design an automated device for scanning objects or persons, size of whom is limited by work area of the scanner. The market currently offers professional systems that are single-purpose and used exclusively for scanning people. However, price of such solution varies within tens of thousands of euros, which is highly limiting for broad-scale use. Our goal is to design an all-purpose device usable with less expensive 3D scanners, thus being more affordable compared to commercial solutions. Such device might aid in expansion of 3D scanning on university grounds but might become more affordable in other fields as well.

\section{3D DIGITIZATION}

Even though we do not often realize it and take it as a matter of course, we live in a digital age. In the streets, we may often see people capturing their moments of joy in a form of photography. The Internet is full of literature that can be downloaded with a few clicks and then read. None of this would be possible without digitization. Digitization can be used to convert information, documents, sounds or objects from physical (analogue) form into digital (electronic) form. This makes work easier, increases productivity, and saves time. After such conversion, we can modify, sort and share the data easily.

Archives full of paper-form information will become history, as data can be "stored" to lunch-box-sized memory media or to cloud storages. In addition to that, digital information does not age and its lifespan is determined by lifespan of the data carrier.

3D digitization of objects is based on scanning individual surface points and capturing their coordinates. This creates a so-called point cloud determining the geometric shape of the given object. Through special software, the point cloud can be used to create a polygonal mesh (see Fig. 1), which can then be transformed to a surface (flat) or volumetric object usable in CAD/CAM applications.

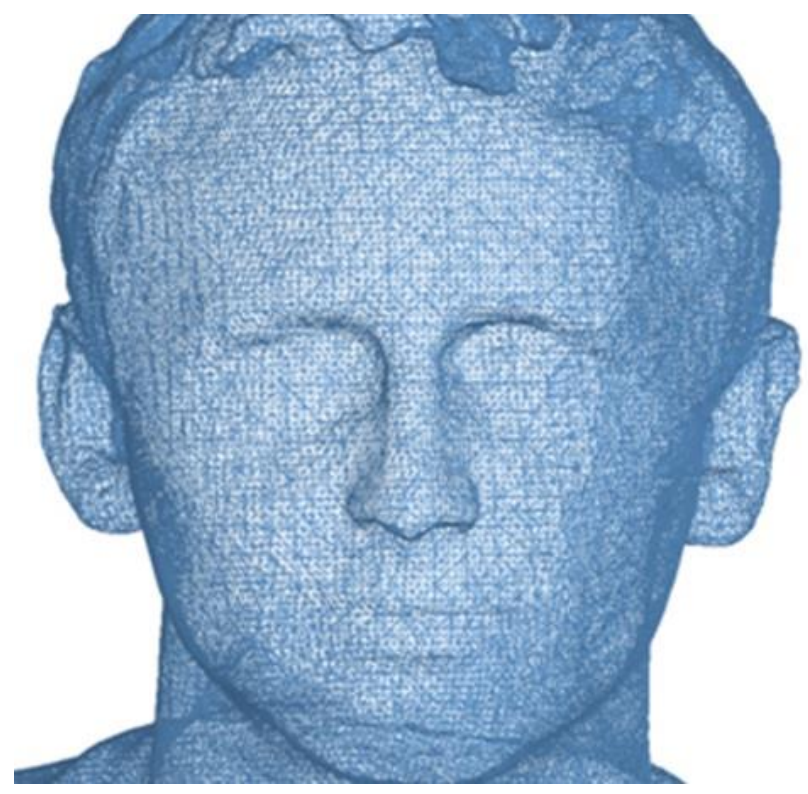

Figure 1. Polygonal mesh of a head from the Sense system

\subsection{Use of 3D digitization of persons}

Human body is one of the important and often used targets of 3D scanning. Through this method, we can capture the whole body or its part. Advantages of obtaining proportions of human body this way are the speed (scanning in 3D scanning room takes only several tens of seconds) and high precision without any necessary contacts with the scanned object. According to the required applications and depending on the used scanner, the model can be represented as a point cloud or polygonal mesh and fitted with colours and textures. 3D scanners can be used to significantly improve the possibilities of precise measurement and visualization of shape, dimensions and surface of human body. Recent improvements in the field of scanning have shown new ways of 3D digitization, particularly in the field of medicine. For example, it can be used to monitor patient's condition such as the course of pregnancy and development of foetus (see Fig. 2). The obtained data can help the practitioners to observe foetus development or identify any future complications.

Photogrammetry and projection of structured light are the primary methods of 3D scanning of bodies. Photogrammetry allows fast 3D scanning process consisting of taking many images of an object at one time and from different angles, then the images are processed using an algorithm to "merge" them together into a 3D model. Photogrammetry technology offers high quality, colour and structured body scanning.

When using structured light systems, a series of linear lighting patterns is cast on an object. The 3D scanner then generates an accurate 3D model using triangulation. This 3D scanning 
technology is highly accurate, but requires the subject to be very still [Lansard 2018]. Nowadays, the market also offers more and more cost-effective scanners with simple controls, making them more available, even to the general public.

In medicine, 3D scanning is used for four fundamental applications [Lansard 2018]:

Epidemiology - use in anthropometric studies (addressing measurement, description and analysis of body parts characterizing growth and structure of body), e.g. studies on development of obesity with children.

Diagnosis - used mostly to treat skin related disorders (bacteria in pores, wrinkles, etc.) and detection of deformations through analysis of patient's body shape in a 3D environment.

Treatment $-3 D$ scanning of patients with severe burns can make the difference between life and death. Precise amount of damaged tissue must be calculated on order to provide the correct amount of medicine.

Monitoring - monitoring of changes to body changes of patients (shape, composition), monitoring during pregnancy or diet.

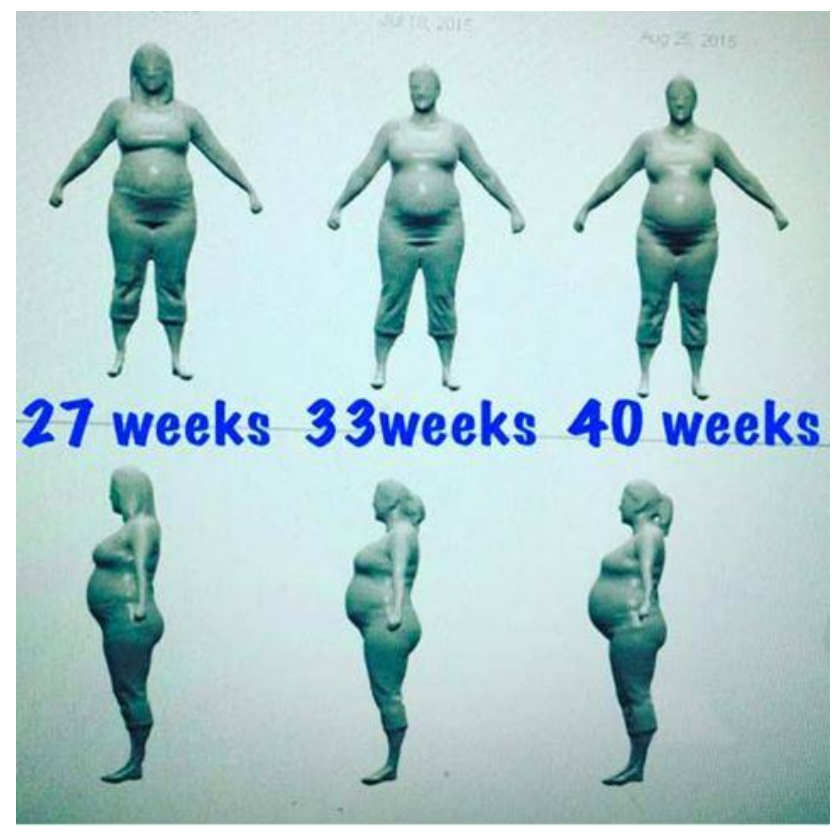

Figure 2. Scanning during pregnancy [Lansard 2018]

3D scanners also brought huge improvements to the field of prosthesis manufacturing, where hand-held scanners are used (see Fig. 3). Advantages of this scanner are its speed, costeffectiveness and high comfort of the patient. In order for the prosthesis to perfectly adapt to the patient's limb, scanning the mounting point is the first step. The 3D model is then used to model a prosthesis and is then additionally modified by software. Prosthesis production is the last step. Rapid Prototyping is broadly used nowadays. Since the resulted prosthesis is "tailored", it is more comfortable, offers a longer lifespan and is more efficient.

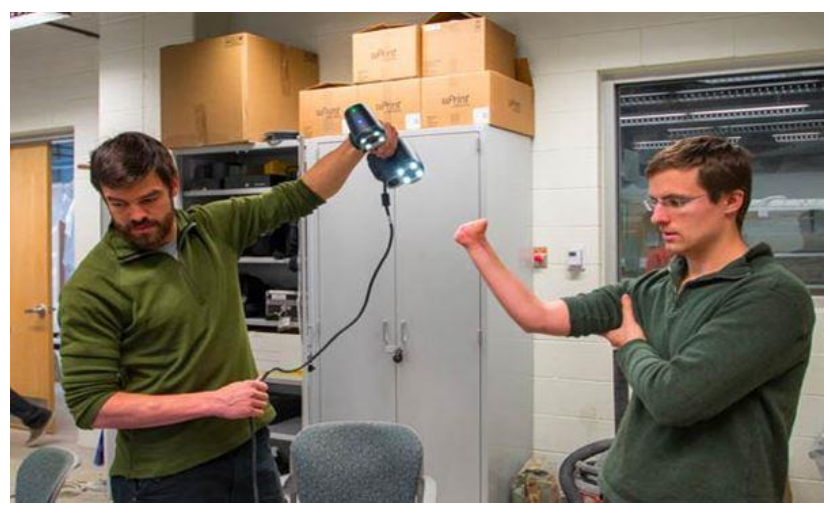

Figure 3. Scanning of limb stump [Lansard 2018]

3D scanning is also used for printing 3D figures. Cabin scanners are very often used to manufacture figures. This type of scanner is very fast and it is therefore not necessary to have the object in fixed position for tens of seconds. The obtained data can be used very easily to print a look-alike 3D figure. The only drawback of these figurines it the high purchase price caused by expensive equipment required to produce the figure.

Recently, an application has been developed which allows using 3D scanner to create a virtual room, where the customers can try out clothes [Lansard 2018]. The concept is based on a simple client account, to which the client uploads an "avatar" representing his or her body. The user is then able virtually try out individual clothes from home without going to the store. This application is highly demanded by Internet stores for the purpose of decreasing costs of returning clothes that do not fit.

Even several fitness centres started using 3D scanners (see Fig. 4). 3D scanners can be used to precisely observe changes to our body and keep reaching our desired goals. Scanners are able to precisely measure the body and observe muscle development and loss of fat. One can be also notified about incorrect body posture. If you store the results of regular scanning, the changes can be observed with time.

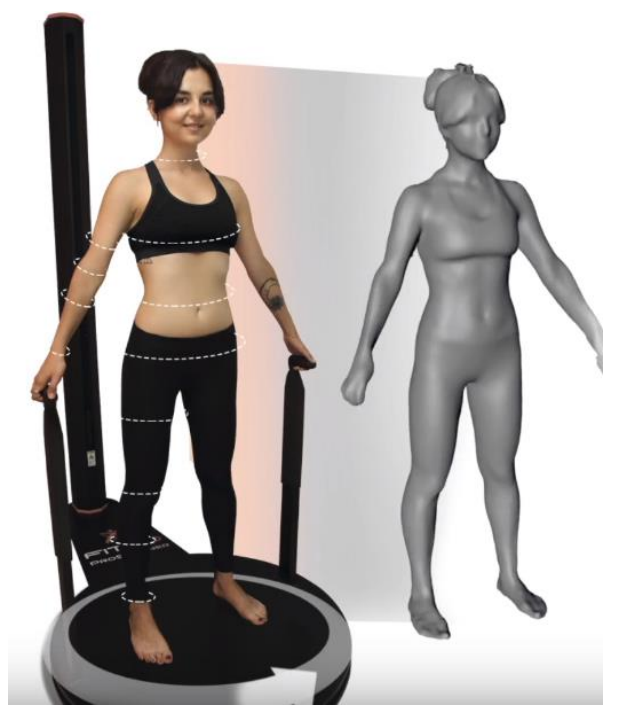

Figure 4. Scanners used in fitness business [Lansard 2018]

3D scanners for human body were used by Krzysztof Kowalski's [llska 2017] team in their project of designing compression products used for external treatment. The scanner has been used to obtain statistical tolerance analysis of human body. Application of 3D scanners in designing compression products is 
also mentioned in [Salleh 2011, Salleh 2012]. In his research, Salleh sought to develop a method for designing customised pressure garments that can be applied onto burn wounds more accurately. The customised pressure garments constructed by means of the developed method (use of a 3D digital image of the wounded body part obtained by a body scanner) have shown a more accurate fit to uniformly exert the design pressure onto the whole wounded area. In his second article, Saleh developed a new method that can be used to construct a customised compression garment for athletes. The garment was generated using 3D data of an athlete's body segment obtained by a noninvasive non-contact measurement system. The research brought a system that allows more than the possibility to design a customised compression garment.

For more information about 3D scanners for human bodies, scanning techniques, and main applications, see also: [Daanen 2018], [Haleem 2019].

\section{METHODS AND MATERIALS}

Design of three-axis scanning device for human body is addressed for example in [Lee 2018]. The research team lead by Miyeon Lee intended to design an inexpensive device that will allow a broad group of scientists to have easy access to 3D data of large objects such as body or clothing. However, they encountered a problem caused by turn-table mechanism design. Design of cabin-based 3D scanner fitted with tens of cameras was being created by a team of authors from university in North Dakota [Straub 2014]. They intended to create 3D models for printing and other applications. This article discusses several possible ways of using the unit and technology.

Another project focused on scanner design is [Grazioso 2017]. Its authors pursue a scanner design that would be used mostly in medicine. The primary goal was to manufacture a device that will allow instant acquisition of structure of human anatomy, while being as non-invasive as possible. The system shows the following key properties: I. instant capture of human model, II. accuracy of the device ranging within $1 \mathrm{~mm}$, III. simple use, IV. possibility to scan with various settings. It is a sophisticated device, which is however quite large and expensive.

Prices of professional scanning devices may reach tens of thousands of euros. These systems are also usually quite large and their relocation may take some time. Therefore, we decided to develop our own design that will be inexpensive and sufficiently mobile. It has been estimated during the research that the final price might be around 1,000 euros.

The design was inspired by construction of Fuboss scanner [Fuboss 2018]. Similarly, it is based on rotating the scanned object and moving in Z-axis (vertical axis) and tilting on B-axis (horizontal axis). The scanned object is rotated by means of a turntable separated from main frame of the moving axis. Meaning that three moving axes can be controlled.

The scanner is fitted to a movable platform with vertical positioning. The axis is designated as $Z$ in Fig. 5 . When starting the scanning process, the platform moves to a reference position secured by $\mathrm{K} 1$ end switch. This begins the scanning process. As soon as the platform reaches the $\mathrm{K} 2$ end switch, the whole process ends. The maximum range of $Z$ axis is $2 \mathrm{~m}$. When scanning smaller persons or objects, the endpoint may be set in the software. MSW-0 micro switch is used as end switch.

B-axis rotation allows tilting of the scanner. Similarly, in the vertical axis, the reference position must be reached before the scanning process starts. That is ensured by IP S-8 inductive sensor. Scanner tilting allows better scanning of areas (surfaces) that are under high angle towards the vertical plane and would not be scanned properly. These are particularly chin and top of the head.

The $\mathrm{C}$ rotation rotates the scanned object to allow scanning from all sides and secure that the resulting model is complete.

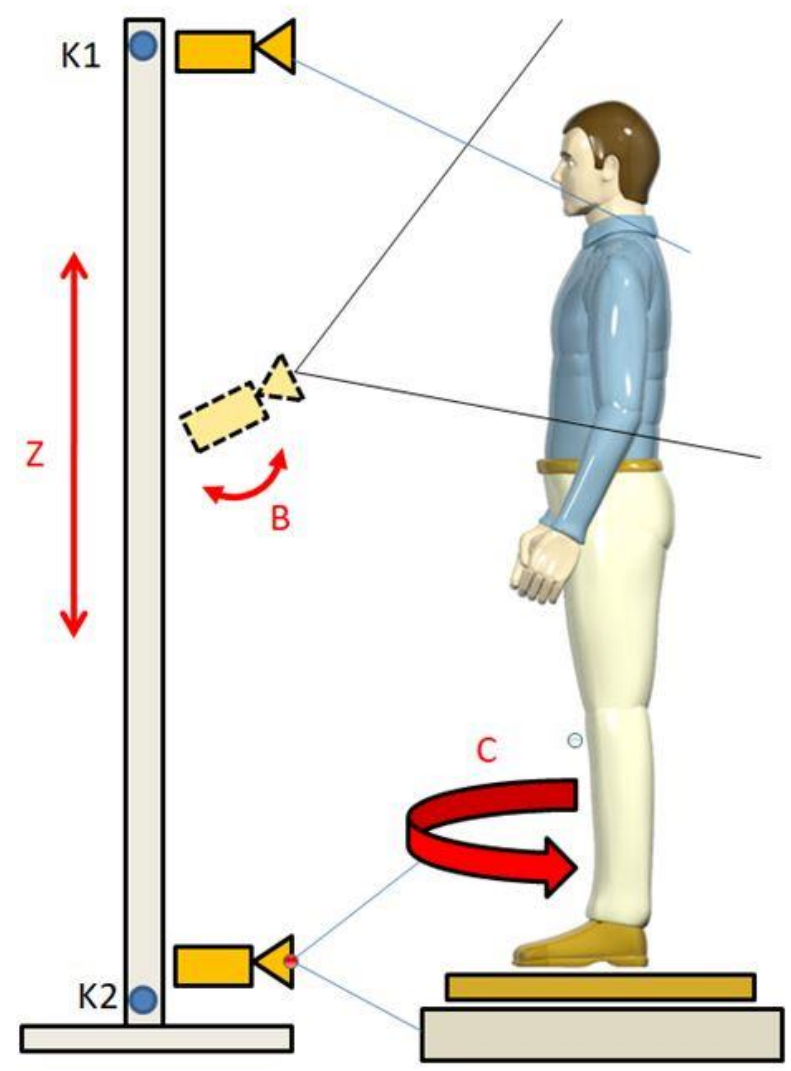

Figure 5. Basic concept of the scanner

\subsection{Frame}

The frame is designed and made of aluminium profiles (see Fig. 6). These profiles allow us to have a stiff but light structure. Manufacturers offer profile bars of various shapes and dimensions. These are easy to connect and combine. For that reason, these profiles were used for small lines, single-purpose machines and hobby devices.

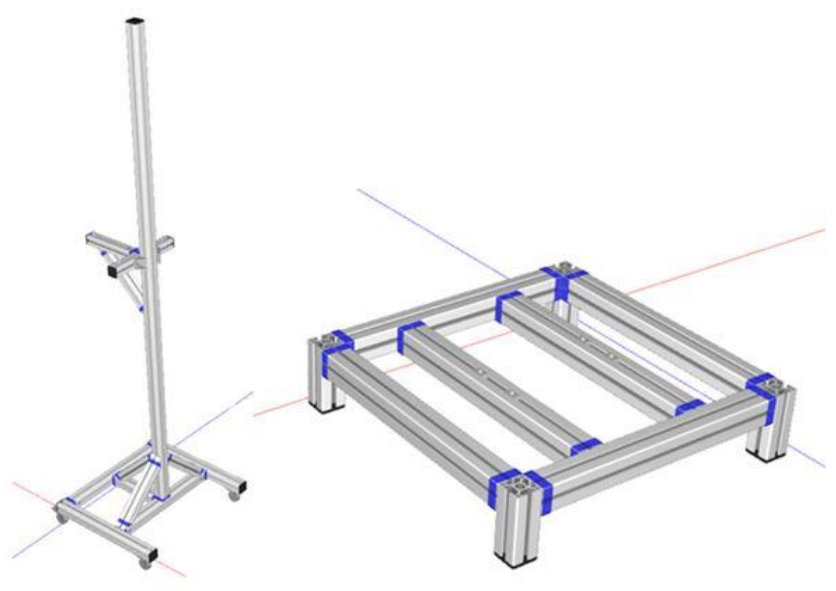

Figure 6. Concept of aluminium profile frame 
The frame was designed in May-CAD software by May-TEX [MayTec 2018]. This company focuses on manufacturing aluminium profiles and its software makes designer's work much easier. All designs can be converted into a STEP model or other formats. The software also estimates the price of the frame, which is beneficial for calculating the estimated price. The frame is comprised of two parts - the base and the stand. The base is used as a support for the stand and is fitted with four wheels, which allow easy handling. Sides of the stand are supported, thus increasing stiffness and stability thereof. There are two aluminium plates on the stand, which are used to fixate guides and the trapezoidal screw. In mid-height of the stand, the aluminium profiles form a "table" used for a laptop or other scanner controls and platform controls.

\subsection{Actuation of frame and table}

Transmission of rotary motion of the motor onto platform motion is ensured by trapezoidal screws made of 12020 steel with plastic nut. Plastic nuts provide adequate accuracy and very simple and maintenance-free operation. The bolt is designed to withstand stress load applied onto it.

Weight of the moving platform is comprised of weight of the scanner, flange and accessories (bolts, scanner rotation motor, etc.). The selected scanner weights $750 \mathrm{~g}$. The remaining weight is given by the structure (Fig. 7). $m=m_{\text {skeneru }}+m_{\text {př́ruba }}+m_{\text {doplňků }}=0,75+1,4+1,5=$
$3,65 \mathrm{~kg} \approx 3,7 \mathrm{~kg}$

Total weight of the moved mass is $3.7 \mathrm{~kg}$.

\begin{tabular}{|l|l|l|l|}
\hline Entered parameters & m & 3.7 & $\mathrm{~kg}$ \\
\hline Weight & $\mathbf{X}_{\max }$ & 2000 & $\mathrm{~mm}$ \\
\hline Stroke & $\mathbf{f}$ & 0.15 & \\
\hline Friction coefficient in bolt & $\mathbf{V}_{\mathbf{p}}$ & 1.3 & $\mathrm{~m} \cdot \mathrm{min}^{-1}$ \\
\hline Velocity & a & 90 & $\circ$ \\
\hline Bolt tilt & t & 0.2 & $\mathrm{~S}$ \\
\hline Bolt acceleration & g & 9.81 & $\mathrm{~m} \cdot \mathrm{s}^{-2}$ \\
\hline Gravity acceleration & E & 210000 & $\mathrm{Mpa}$ \\
\hline Modulus of elasticity & K & 3 & \\
\hline Safety & $\mathbf{f u}$ & 2 & \\
\hline Seating type & A & 3.927 & \\
\hline Screw seating coefficient & P & 7800 & $\mathrm{~kg} \cdot \mathrm{m}^{-3}$ \\
\hline Screw material density & & &
\end{tabular}

Table 1. Selected parameters

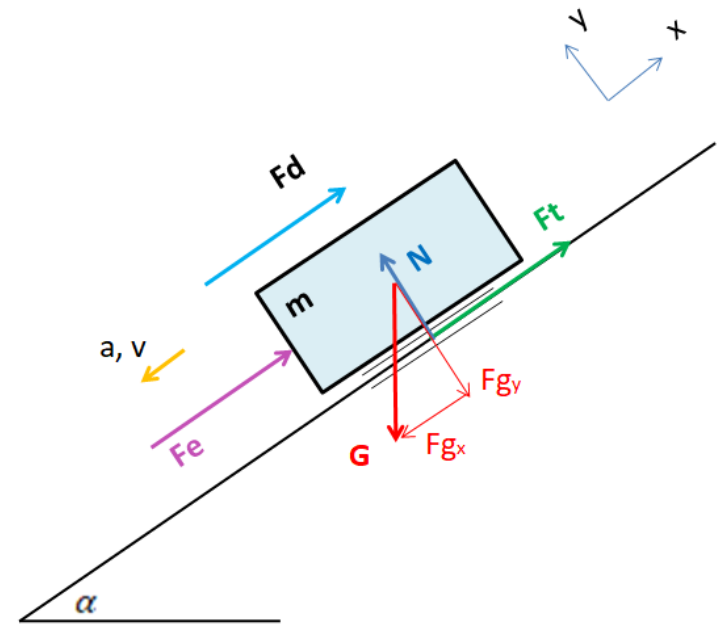

Figure 7. Forces applied to the bolt

Loading force

$\mathrm{F}_{\mathrm{zat}}=\mathrm{F}_{\mathrm{e}}+\mathrm{F}_{\mathrm{T}}+\left|\mathrm{F}_{\mathrm{gx}}\right|+\mathrm{F}_{\mathrm{d}}$

There will be no external force stressing the bolt, since no external forces apply on the portal

Gravitational component of the force

$\mathrm{F}_{\mathrm{gx}}=\mathrm{m} \cdot \mathrm{g} \cdot \sin \alpha=3,7 \cdot 9,81 \cdot \sin 90=36,3 \mathrm{~N}$

Friction force $F_{t}$ is zero because the angle $\alpha$ is $90^{\circ}$.

Dynamic force

$\mathrm{F}_{\mathrm{d}}=\mathrm{m} \cdot \mathrm{a}=\mathrm{m} \cdot \frac{\mathrm{v}}{\mathrm{t}}=3,7 \cdot \frac{1,3}{60 \cdot 0,2}=0,4 \mathrm{~N}$

$\mathrm{F}_{\mathrm{zat}}=\mathrm{F}_{\mathrm{e}}+\mathrm{F}_{\mathrm{T}}+\left|\mathrm{F}_{\mathrm{gx}}\right|+\mathrm{F}_{\mathrm{d}}=0+0+36,3+0,4 \approx 36,7 \mathrm{~N}$

This force must not exceed the permitted force when loading bolt to strut.

$\mathrm{F}_{\mathrm{zat}} \leq \frac{\mathrm{f}_{\mathrm{u}} \cdot \pi^{2} \cdot \mathrm{E} \cdot \mathrm{I}}{\mathrm{x}_{\max }^{2} \cdot \mathrm{k}}$

This condition can be used to determine minimum bolt diameter using quadratic section modulus $I$.

$\mathrm{d}=\sqrt[4]{\frac{64 \cdot \mathrm{x}_{\text {max }}^{2} \cdot \mathrm{k} \cdot \mathrm{F}_{\mathrm{zat}}}{\pi^{3} \cdot \mathrm{f}_{\mathrm{u}} \cdot \mathrm{E}}}=\sqrt[4]{\frac{64 \cdot 2000^{2} \cdot 3 \cdot 36,7}{\pi^{3} \cdot 2 \cdot 210000}}=6,82 \mathrm{~mm}$

We selected a TR -1608 bolt

Rise: $\mathrm{s}=8 \mathrm{~mm} /$ rotation Diameter: $\mathrm{d}_{\mathrm{n}}=16 \mathrm{~mm}$

It is also imperative to check whether the critical speed has not been exceeded. Otherwise, the bolt might start to vibrate.

$\mathrm{n}_{\mathrm{krit}}=\frac{60 \cdot 10^{6} \cdot \lambda^{2}}{2 \cdot \pi \cdot x_{\max }^{2}} \cdot \sqrt{\frac{\mathrm{E} \cdot \mathrm{I}}{\rho \cdot \mathrm{A}}}$ 
Calculation will provide

$\mathrm{n}_{\mathrm{krit}}=\frac{60 \cdot 10^{6} \cdot 3.927^{2}}{2 \cdot \pi \cdot 2^{2}} \cdot \sqrt{\frac{2,1 \cdot 10^{5} \cdot 3217}{7800 \cdot 201}}=764,2 \mathrm{rpm}$

Actual speed

$\mathrm{n}=\frac{\mathrm{vp}}{\mathrm{s}}=\frac{21,66}{8}=2,7 \mathrm{rev} \cdot \mathrm{s}^{-1}=162 \mathrm{rpm}$

$\mathrm{n}<\mathrm{n}_{\text {krit }}$

This condition is met and there is no risk of screw oscillation.

Actuation with stepper motors was chosen. The reason for that is simple control and lower price. The motor was designed in accordance with [Marek 2015]. However, detailed calculation cannot be provided due to the extent thereof. Firstly, a required motor torque was determined from statistic perspective (determination of shift and technological forces). Such calculation was based on force relationships (load component torque, torque load from the friction forces of moving masses, torque load from friction forces in bearing, torque load from axial force offset), effectiveness and gearing ratio has been considered as well. The design was also considered from the perspective of dynamics, where the total reduced load torque is important. The resulting load torque is given by a sum of static and dynamic torque. The value would approximate $0.14 \mathrm{Nm}$. Based on the obtained values, we decided to use $42 \mathrm{HSO} 3$ bipolar stepper motor. Its torque characteristics is shown in Fig. 8. Torque of the motor is transmitted onto the trapezoidal screw through a belt drive.

Actuation of rotational axis of the turntable is ensured by a stepper motor as well. The properties were calculated for inertia torque generated by a $182 \mathrm{~cm}$-high man with a weight of $90 \mathrm{~kg}$. Based on inertia torque generated by the load, friction and gear ratios (belt drive is located between the motor and drive shaft), the minimum required torque is $2.15 \mathrm{Nm}$. Therefore, $86 \mathrm{HS} 35$ motor was selected.

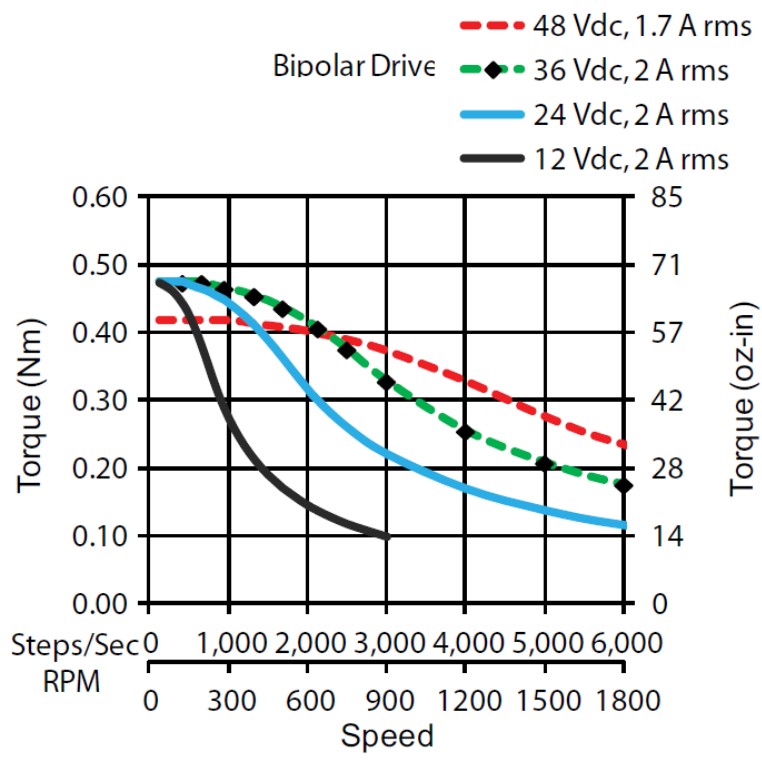

Figure 8. Torque characteristics of 42HS03 motor [PBC linear 2018]

\subsection{Scanner}

Firstly, we tried to use Kinect. It is a scanner sold as accessories to Xbox consoles. The scanner is basically an IR (infra-red) projector projecting a grid of IR points, which is then captured with IR CMOS camera sensitive to IR bands only. The system also features an RGB camera, which is capable of mapping object colours onto a 3D model. Kinect costs around 100 euros. Unfortunately, Microsoft stopped supporting this device, therefore any updates or future support will be unavailable. Proper operation of the system requires a third-party software, price of which is an additional $€ 100$. There are other problems when using the device on Windows 10 systems. The communication between the scanner and the PC was very problematic due to the unavailability of the necessary drivers. Therefore, we decided to use a different scanner for testing purposes.

Namely, we used a simple hand scanner called Sense, manufactured by 3D Systems (see Fig. 9). This system was chosen mainly due to its affordability and good experience with scanning the human body. The manufacturer offers a possibility to scan a head or the whole body in the control SW. The scanner operates by determining the distance of structured light projection. However, such structured light is in IR spectrum and is therefore not visible by the human eye. As in the case of Kinect, the Sense system features an IR projector, IR detector and a camera that senses colours of the object. This solution costs around $€ 400$. The price already includes manufacturerprovided SW which can be readily used to control the scanner and offers simple data processing, including the creation of point cloud, or polygonal mesh with possible export to formats such as VRML, STL, OBJ, and PLY. However, as already mentioned, the scanning platform is versatile, and any other systems meeting the weight criteria may be used. Therefore, we are not limited to using this scanner only.

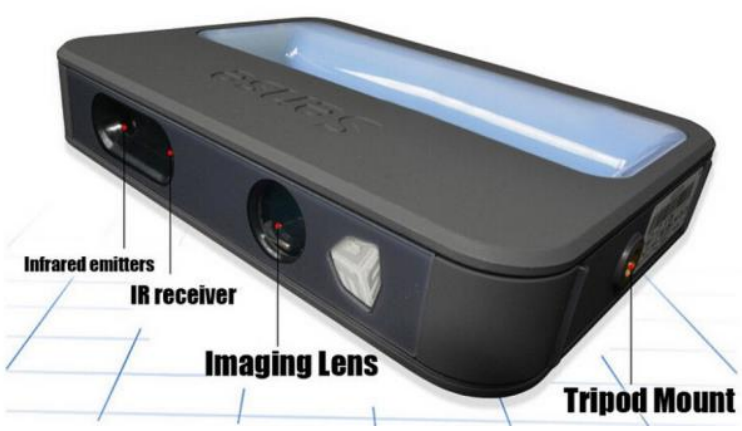

Figure 9. Sense scanner [AliExpress 2018]

The technical parameters indicated by the manufacturer are given in the Table 2.

\begin{tabular}{|l|c|c|}
\hline \multicolumn{1}{|c|}{ Parameter } & Kinect & Sense \\
\hline Operating range [m] & $0.5-4.5$ & $0.2-1.6(3)$ \\
\hline Depth image size [px] & $512 \times 424$ & $640 \times 480$ \\
\hline Colour image size [px] & $1920 \times 1080$ & $1920 \times 1080$ \\
\hline X/Y resolution @ 0.5m [mm] & - & 0.9 \\
\hline Depth resolution @ 0.5m [mm] & - & 1 \\
\hline
\end{tabular}

Table 2. Technical specifications of the scanner Kinect and Sense [3D systems]. 
Since the manufacturer does not provide scanning accuracy data (according to some studies, the Kinect system ranges within centimetres [Khoshelham 2012], [Lachat 2015]), a comparison experiment regarding quality and accuracy of the said systems was made before a final decision regarding which scanner is to be used was made. No standardized methodology was intentionally used to evaluate the accuracy, since the aim of the research was not to evaluate the accuracy of the scanners, but primarily to design a scanning platform (various scanners can be mounted on the platform). The test is intended only to point out the significant qualitative differences between the two systems. During the test, an object was scanned with both scanners under identical light conditions. The obtained scans were compared with the model scanned by the professional ATOS TripleScan system, which offers a certified measurement accuracy of 0.01 $\mathrm{mm}$. The scan quality differs at first glance, as can be seen in the colour deviation maps (see Fig. 10).

The surface scanned by the Sense scanner is smooth and the scanner managed to capture high level of details. Maximum deviations range up to $1 \mathrm{~mm}$, which is satisfactory for our purposes. In comparison, the Kinect system lacks the ability to capture details and the dimensional accuracy is roughly twofold.

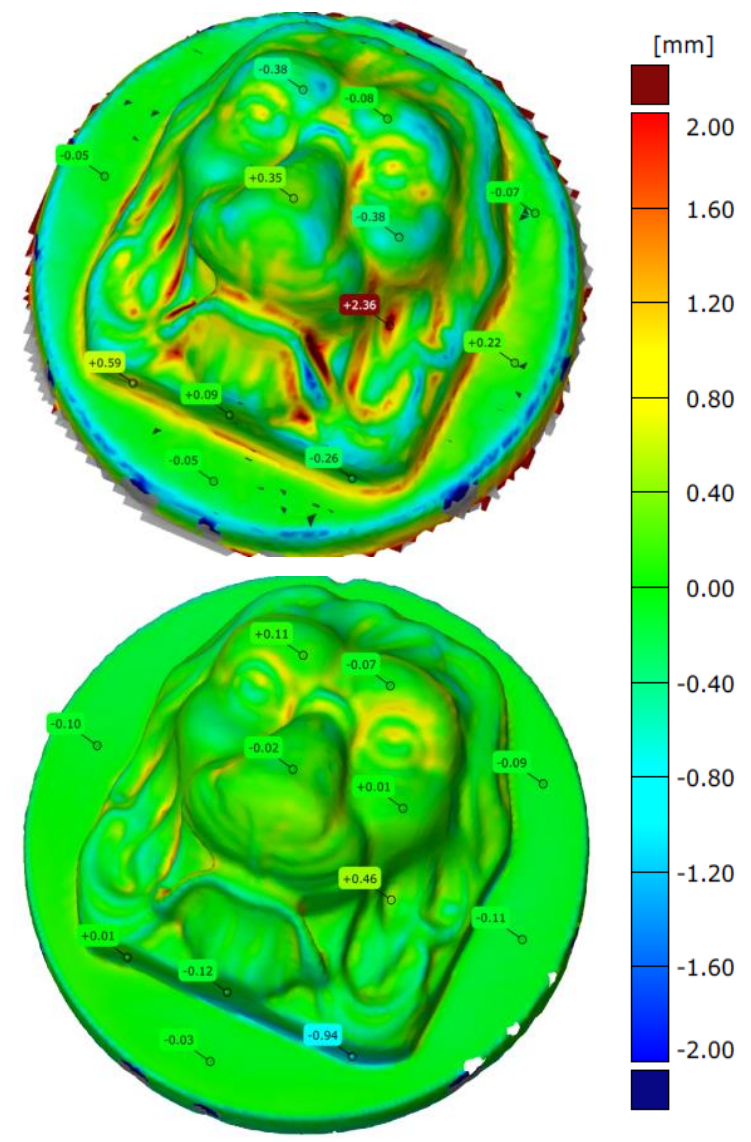

Figure 10. Deviation map of Kinect (top) and Sense (bottom) scanners

\subsection{Guide and platform}

The Guide is secured with two unsupported rods, ends of which are fitted with clamping handles. These additional guides are supposed to provide additional stability and stiffness to the system. Each of these two rods is used to move two linear sets. It consists of a ball sheathe secured by a retaining ring in a housing. The platform is then attached to the housing, which is used to fasten the scanner. The selected rod diameter is $16 \mathrm{~mm}$.
The platform is used to attach the tilting components and the scanner assembly. This platform is mounted to the sheathe of trapezoidal nut by means of countersunk screws. In order to minimize the stress applied onto the stepper motor, the part was made of aluminium and milled for the purpose of weight reduction (see Fig. 11). Due to the selected shape and openings, we managed to reduce the weight by $34 \%$. With that, we could use a smaller stepper motor.

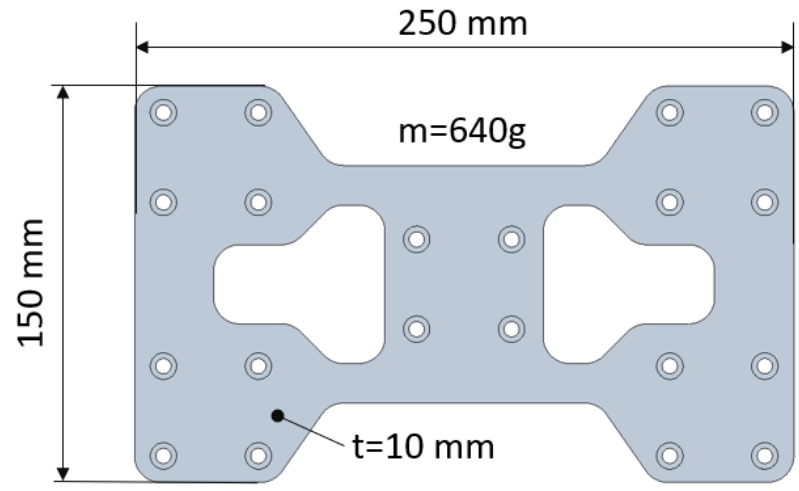

Figure 11. Part with reduced weight

Two L-shaped supporting elements were mounted to the platform (Fig. 12 -yellow parts). These elements are used to seat the tilting plate and are, same as the plate, manufactured by means of Rapid Prototyping method, specifically the FDM technology (Fused Deposit Modelling). The tilted plate is seated from the left side into the housing, which is fitted with a plastic shim. This improves the surface quality and decreases friction coefficient. On the right side, the plate is attached to stepper motor shaft and secured with locking screw. Additionally, the tilting plate can be fitted with a LED light, which can be used to light the scanned object in case of bad light conditions.

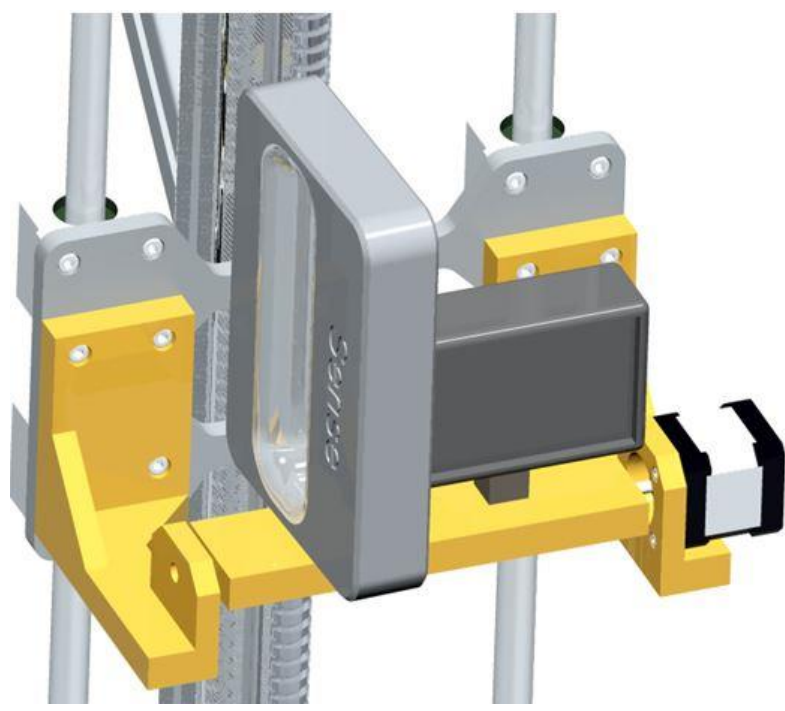

Figure 12. Final design of the platform 

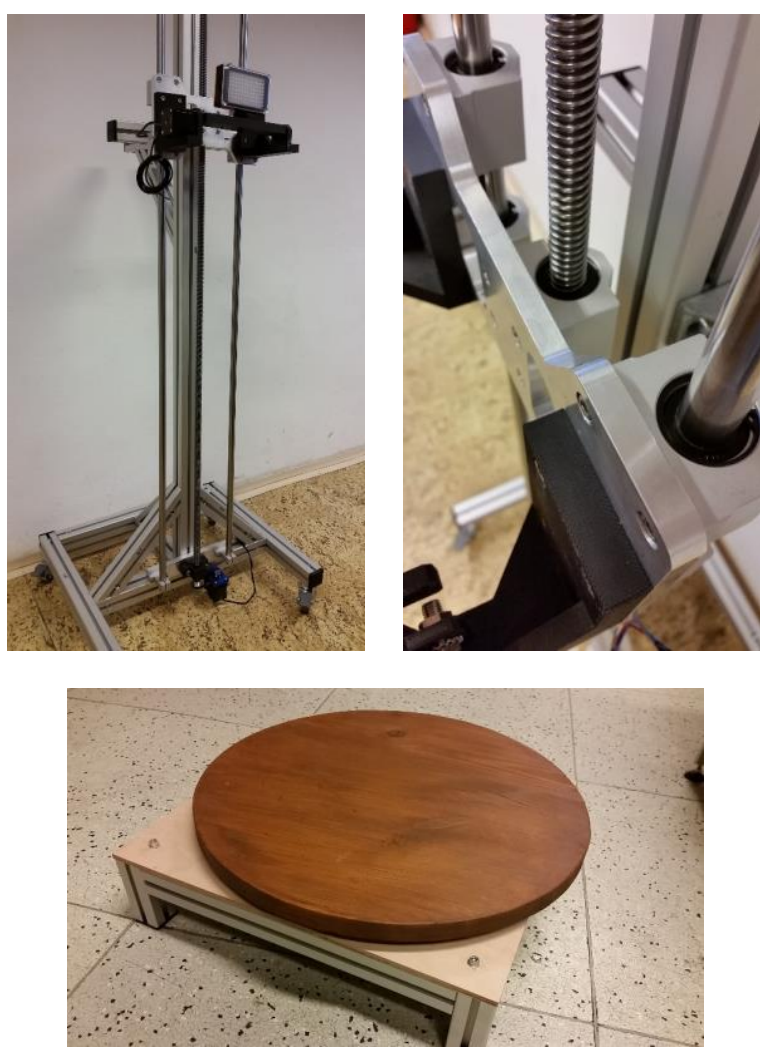

Figure 13. Photos of the manufactured platform

\subsection{Control system}

The Arduino UNO development platform was selected as a means of control of stepper motors and setting of the scan parameters (scanner feed speed, scan area, etc.). This allows a wide range of shields (plug-ins) and other separate peripherals to be connected. The motherboard is extended with CNC shield specially designed for controlling stepper motors. This combination ensures a sufficient number of inputs and outputs and allows the drives to be controlled efficiently.

The movement of the vertical feed (Z-axis) and the scanner rotation (B-axis) is secured by $42 \mathrm{HSO} 3$ (NEMA 17) motors. These two stepper motors are directly connected to the CNC shield, which is further fitted with two drivers for Pro Reprap 3DA4988 stepper motors. This CNC shield is then connected directly to the Arduino UNO motherboard. In the first phase, the control was carried out by means of the grblControl program. You can set the number of steps, direction of rotation and feed rate.

\subsection{Price calculation}

Unfortunately, the resulting price (Table 3 ) exceeded the estimated one by less than $€ 400$. The total price of all purchased components (such as Arduino Uno, stepper motors, Scanner frame etc.) was $€ 913$. Another important item was the price of printing of the 3D parts. It is a very fast and effective way of manufacturing prototypes. However, price of the technology strongly exceeds the price of conventional machining methods.
In case of higher number of manufactured parts, it would be wise to use standard manufacturing methods. It is anticipated that doing so would reduce price of these parts by at least $50 \%$. The prices include VAT. Price of the scanner has not been included, since the mounting platform can be fitted with multiple types of scanners.

Despite exceeding the estimated cost, the price is still significantly lower than in the case of commercial systems. Thanks to the simplicity, high mobility and all-purpose nature of the designed solution, the 3D scanning platform has a major potential in the market.

\section{CONCLUSION}

The goal of this project was to design a system that will be inexpensive and that will offer similar features as some of the professional systems. Despite deviating from the plan, this device is still considerably cheaper and provides a certain flexibility to the users compared to commercial scanners. This is ensured by a multifunctional platform that can be fitted with any flange for any scanner or device. The scanner holder can also be fitted with a LED light to secure uniform lighting of the scanned scene. Also, the whole system can be disassembled in order to increase its mobility. Operators can reassemble the disassembled system in 15 minutes and start scanning.

Another advantage of this solution is its open nature and variability. The system allows not only scanning of the entire human body, but also fast digitization of its parts (head, torso, limbs). It can also be used for automatic scanning of static objects up to $2 \mathrm{~m}$ in height and with up to approx. $50 \mathrm{~cm}$ in diameter (e.g. sculptures, plaster and clay designs, etc.). The first tests were carried out in semi-automatic mode, in the entire scanning range, and the rate of each axis were tested. From a mechanical point of view, the system works as expected and the scanning quality corresponds with the capabilities of the Sense 3D scanner used. The research and experimental activities will continue. The plan is to test other scanning systems (including photogrammetry) and, above all, to develop a user-friendly software for controlling the entire platform. In a control panel it will be possible to select the size of the scanned object and control the rates and ranges of the individual motion axes according to the type of the scanned object. It is also planned to automatically check the error rate of the scanning process and, if necessary, to automatically repeat the operation.

\section{ACKNOWLEDGEMENT}

This publication was written at the Technical University of Liberec, Faculty of Mechanical Engineering with the support of the Institutional Endowment for the Long-Term Conceptual Development of Research Institutes, as provided by the Ministry of Education, Youth and Sports of the Czech Republic in the year 2019.

\begin{tabular}{|l|r|}
\hline \multicolumn{1}{|c|}{ Item name } & \multicolumn{1}{c|}{ Total } \\
\hline Purchased components & $913.00 €$ \\
\hline Screw machining & $85.00 €$ \\
\hline Printing of components & $400.00 €$ \\
\hline Total price & $\mathbf{1 3 9 8 . 0 0 €}$ \\
\hline
\end{tabular}

Table 3. Price calculation 


\section{REFERENCES}

[3D systems] Sense 3D Scanner | Technical Specifications | 3D Systems. [online]. [date of citing 29/08/2019]. Available from <https://www.3dsystems.com/shop/sense/techspecs>.

[AliExpress 2018] AliExpress, Sense 3D handy 3D scanner, January 2019 [online]. [date of citing 2019-01-30]. Available from <https://www.aliexpress.com/item/1967615179.html >.

[Daanen 2018] Daanen, H. A. M. and A.Psikuta. 3D Body Scanning. Automation in Garment Manufacturing, Elsevier, 2018, pp. 237-52. ISBN 978-0-08-101211-6. DOI 10.1016/B9780-08-101211-6.00010-0.

[Fuboss 2018] The 3D Body Scanning Within Your Reach. January 2019 [online]. [date of citing 30/01/2019]. Available from <htps://www.fuboss.es/>.

[Grazioso 2017] Grazioso, S., et al. Design and development of a novel body scanning system for healthcare applications. International Journal on Interactive Design and Manufacturing (IJIDeM). May 2018. Vol. 12, no. 2, pp 611-620. ISSN 19552505. DOI 10.1007/s12008-017-0425-9.

[Haleem 2019] Haleem, A. and M. Javaid. 3D Scanning Applications in Medical Field: A Literature-Based Review. Clinical Epidemiology and Global Health. June 2019. Vol. 7, No. 2, pp. 199-210. ISSN 2213-3984. DOI 10.1016/j.cegh.2018.05.006.

[Ilska 2017] Ilska, A., et al. Using a 3D Body Scanner in Designing Compression Products Supporting External Treatment. Fibres and Textiles in Eastern Europe. 31 October 2017. Vol. 25, no. 0, pp 107-112. DOI 10.5604/01.3001.0010.4636.

[Khoshelham 2012] Khoshelham, K. and S.O. Elberink. Accuracy and Resolution of Kinect Depth Data for Indoor Mapping Applications. Sensors (Basel, Switzerland). Feb. 2012. Vol. 12, no. 2, pp 1437-1454. ISSN 1424-8220. DOI https://doi.org/10.3390/s120201437

[Lachat 2015] Lachat, E., et al. Assessment and Calibration of a RGB-D Camera (Kinect v2 Sensor) Towards a Potential Use for Close-Range 3D Modeling. Remote Sensing. Oct. 2015. Vol. 7, no. 10 , pp. 13070-97. ISSN 2072-4292. DOI 10.3390/rs71013070.
[Lansard 2018] Lansard, M. 3D body scanning, full body scanning and human body 3D scanners. July 5, 2018 [online]. [date of citing 30/01/2019]. Available from <https://www.aniwaa.com/3d-body-scanning>.

[Lee 2017] Lee, M., et al. Development of low cost threedimensional body scanner using depth perception camera. International Journal of Clothing Science and Technology. 6 November 2017. Vol. 29, no. 6, pp 857-867. ISSN 0955-6222. DOI 10.1108/IJCST-02-2017-0010.

[Maly 2018] Maly, V. Draft design solution for automated 3D scanning people. Liberec: Technical University of Liberec. Department of Mechanical Engineering, 2018 (in Czech).

[Marek 2015] Marek, J. et al. Design of CNC machine tools: peer-reviewed monograph. Prague: MM Publishing. MM industrial spectrum, special issue, 2015. ISBN 978-80-260-86376.

[Maytec 2018] Maytec. Aluminium Systemtechnik , Maycad. Januar 2019 [online]. [date of citing 2019-01-30]. Available from <https://maytec.com.de>.

[PBC linear 2018] PBC linear. Stepper Motor NEMA 17. [online]. [date of citing 2019-01-30]. Available from <http://www.pbclinear.com/Download/DataSheet/StepperMotor-Support-Document.pdf $>$.

[Salleh 2011] Salleh, M., N., B., et al. Customised Pressure Garment Development by Using 3D Scanned Body Image. Research Journal of Textile and Apparel. November 2011. Vol. 15, no. 4, pp 9-18. ISSN 1560-6074. DOI 10.1108/RJTA-15-042011-B002.

[Salleh 2012] Salleh, M,N. et al., Development of a flexible customized compression garment design system. In: K. Warwick, ed. The 2012 International Conference on Advanced Mechatronic Systems, Tokyo, Japan, 18-21 September 2012, IEEE, ISBN 978-0-9555293-8-2.

[Straub 2014] Straub, J. and Kerlin, S. Development of a Large, Low-Cost, Instant 3D Scanner. Technologies. 20 May 2014. Vol. 2, no. 2, pp 76-95. ISSN 2227-7080. DOI

$10.3390 /$ technologies 2020076 .

\section{CONTACT}

Ing. Radomir Mendricky, Ph.D.

Technical University of Liberec

Faculty of Mechanical Engineering

Department of Manufacturing Systems and Automatization

Studentska 2, 46117 Liberec 1, Czech Republic

+420485353356

radomir.mendricky@tul.cz

www.ksa.tul.cz 